\title{
What triggered massive hemoptysis in Goodpasture syndrome with isolated pulmonary involvement in a 14-year-old boy?
}

\author{
Tuğba Şişmanlar-Eyüboğlu¹, Ayşe Tana Aslan², Deniz Gezgin-Yıldırım³, Necla Buyan, \\ Serhat Emeksiz ${ }^{5}$, Gökhan Kalkan ${ }^{5}$ \\ ${ }^{1}$ Department of Pediatric Pulmonology, Dr Sami Ulus Maternity and Children Research and Training Hospital; Departments \\ of ${ }^{2}$ Pediatric Pulmonology, ${ }^{3}$ Pediatric Rheumatology, ${ }^{4}$ Pediatric Nephrology and ${ }^{5}$ Pediatric Intensive Care Unit, Gazi \\ University Faculty of Medicine, Ankara, Turkey. E-mail: aysetugbapp@gmail.com \\ Received: 5th December 2017, Revised: 27th June 2018, 19th July 2018, Accepted: 25th July 2018
}

SUMMARY: Şişmanlar-Eyüboğlu T, Aslan AT, Gezgin-Yıldırım D, Buyan N, Emeksiz S, Kalkan G. What triggered massive hemoptysis in Goodpasture syndrome with isolated pulmonary involvement in a 14-year-old boy? Turk J Pediatr 2019; 61: 611-614.

Goodpasture syndrome is a rare, autoimmune disease associated with the development of antibodies against a specific antigen both in glomerular basement membrane and alveolar basement membrane. It is very rare in childhood and its incidence is not known. Although the mechanism of the disease is the same in lung as in the kidney, sometimes it presents with involvement of only one organ. Pulmonary involvement may be lifethreatening in patients with massive hemoptysis. Herein we report a 14-yearold boy with isolated lung involvement. He had massive hemoptysis following bronchoscopy and recovered succesfully with treatment.

Key words: bronchoscopy, child, Goodpasture syndrome, massive hemoptysis, smoking.

Goodpasture syndrome (GS) is a rare, autoimmune disease in which circulating antibodies are directed against a specific antigen intrinsic to the glomerular basement membrane and alveolar basement membrane. It has a frequency of $0.5-1$ case per million/ year but it is much rarer in childhood and typically presents with the syndrome of glomerulonephritis and pulmonary hemorrhage. ${ }^{1}$ Whereas $60-80 \%$ of patients with GS have both pulmonary and renal involvement, $20-40 \%$ of patients have only kidney involvement and less than $10 \%$ of patients have only pulmonary involvement. ${ }^{2}$ Pulmonary symptoms include cough, chest pain, hemoptysis and hypoxia. ${ }^{1}$ Diffuse alveolar hemorrhage may be life threatening. Usually bilateral, symmetric perihilar, and bibasilar parenchymal consolidations may present on chest $\mathrm{x}$-ray and thorax computerized tomography (CT). ${ }^{3}$ The gold standard for diagnosis of GS is a kidney biopsy, however it is not required in life threatening and prompt treatment demanding cases. In suspected cases, it would be useful to detect circulating anti-glomerular basement antibodies (AntiGBM) by ELISA. In addition, monitorizing the quantitative titers of Anti-GBM is useful for following the disease activity. Finally, in GS patients, there is a genetic predisposition for human leukocyte antigen (HLA) DR $15 .{ }^{4}$

Diagnosis might be difficult in patients with atypical presentations. Herein we report a 14-year-old boy with minor hemoptysis for a duration of two months. He had pulmonary involvement without renal disease and massive hemoptysis following bronchoscopy.

\section{Case Report}

A 14-year-old boy with minor hemoptysis for two months was referred to the pediatric pulmonology department for further investigation. He had no history related to infection, fever, malaise, cough, weight loss, 


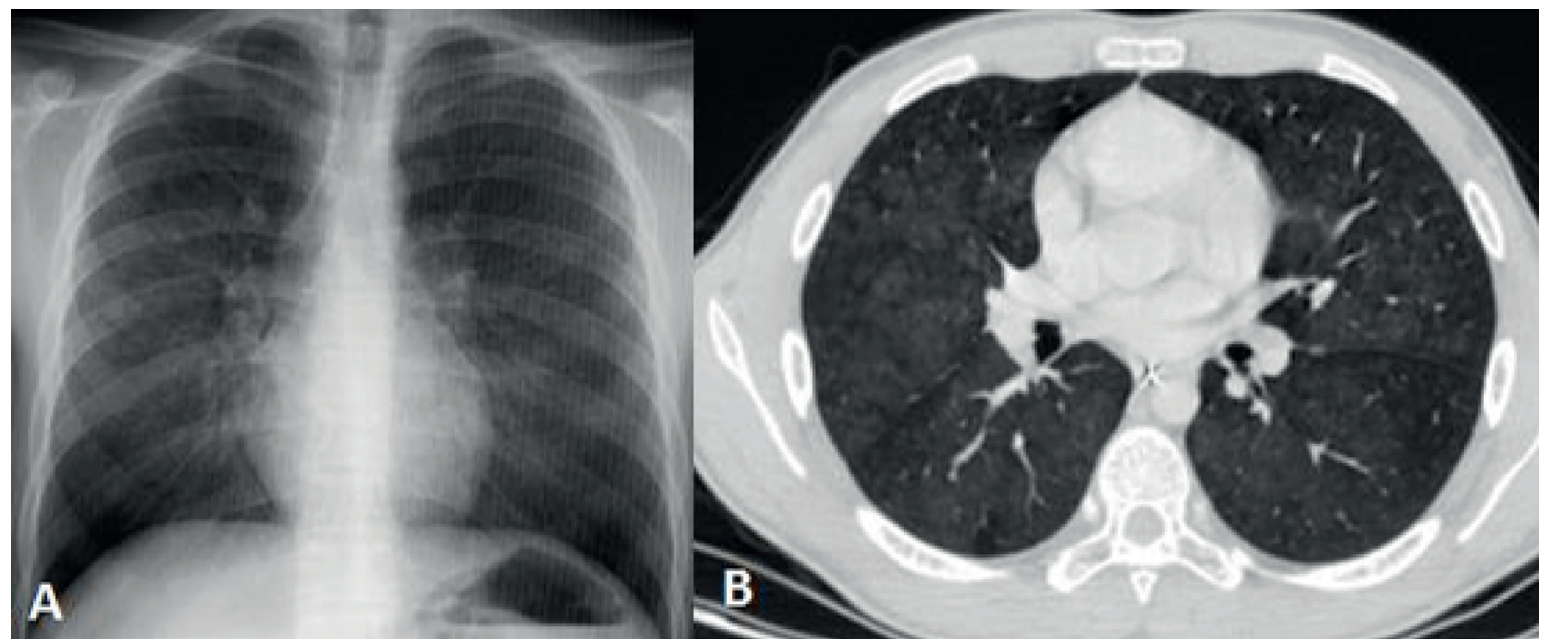

Fig. 1. A) Patchy alveolar infiltrations on both lungs on chest x-ray, B) on thorax CT.

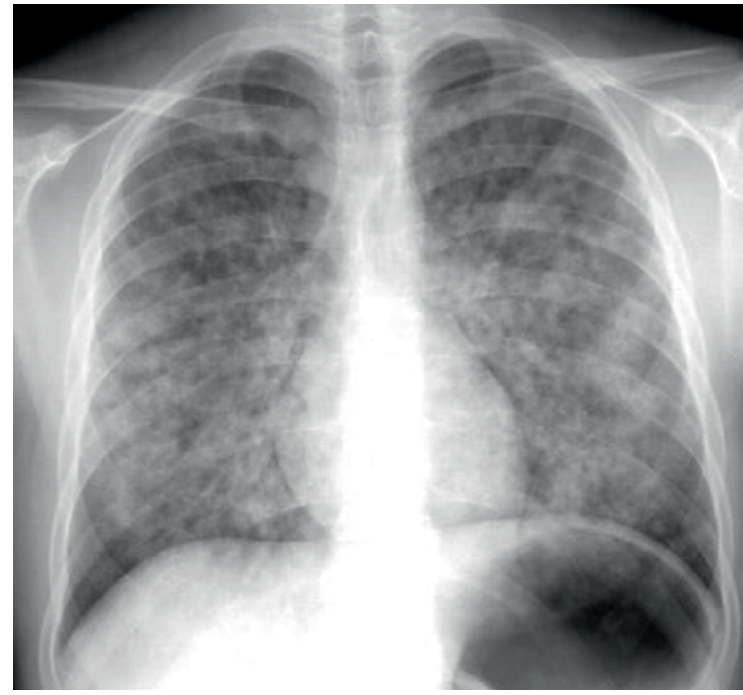

Fig. 2. Diffuse consolidation on both lungs compatible with diffuse hemorrhage.

joint pain, joint swelling, rash, skin eruption, photosensitivity, hair loss or medication use. He had been smoking for the past two years (1 pack-year). Physical examination was normal and there was no hypoxia. Patchy alveolar infiltrations were present on both chest X-ray and thorax CT (Fig. 1). His blood panel showed hemoglobin at $13.8 \mathrm{~g} / \mathrm{dl}$, total white cell count at $12.2 \times 10^{3} / \mu \mathrm{L}$, and platelets at $308 \times 10^{3} / \mu \mathrm{L}$, whereas blood coagulation tests were normal and renal function tests were within normal limits. Acute phase reactants were negative. In immunological workup, Anti-nuclear antibody (ANA), ANA profile, Anti neutrophil cytoplasmic antibody (ANCA) profile, Anti-phospholipid antibodies and

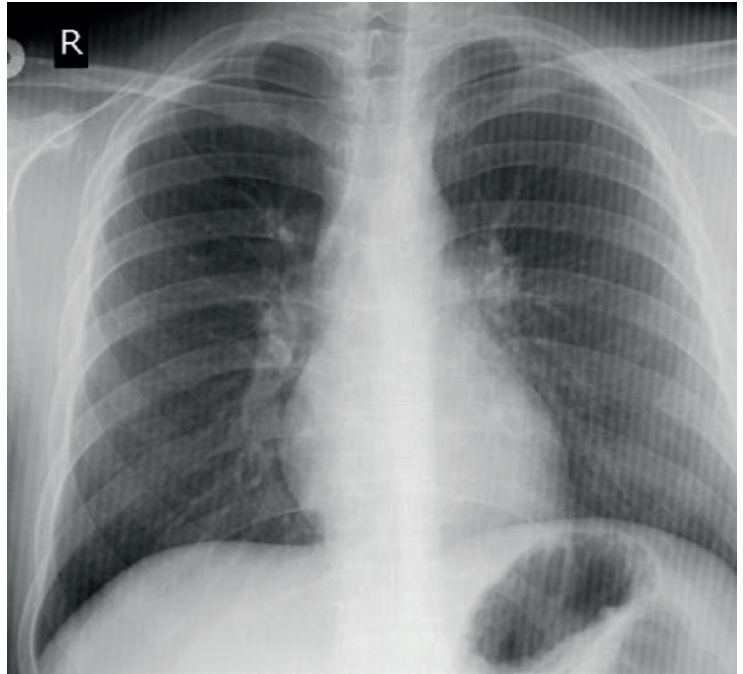

Fig. 3. Normal chest x-ray.

complement levels were all normal, while AntiGBM antibodies were positive. In addition, HLA DR 15 positivity was detected. But he had no proteinuria or hematuria. Uneventful bronchoscopy was performed for diagnosis and acute hemorrhage was not observed. Bronchoalveolar lavage fluid was clear and hemosiderin-laden macrophages were detected. Patient was stable and control chest $\mathrm{x}$-ray to rule out a pneumothorax was normal after bronchoscopy. However, 18 hours after bronchoscopy, the patient exhibited massive hemoptysis. He had tachycardia, tachypnea, pallor and cyanosis. Physical examination revealed common crackles on both lungs with marked retractions. Hemoglobin decreased 
to $6.8 \mathrm{~g} / \mathrm{dl}$. Oxygen supplementation was started, he received a blood transfusion and he was transferred to the intensive care unit. Chest x-ray was compatible with diffuse alveolar hemorrhage (Fig. 2). He was intubated due to respiratory failure and ventilated with high positive end expiratory pressure (PEEP). Kidney biopsy was not performed owing to rapidly progressive life threatening course, also there was no renal findings. Pulse steroid, cyclophosphamide and plasmapheresis treatment were started immediately. Plasmapheresis was performed daily for the first five days then every other day for six treatments. He was extubated on the 5 th day. Treatment was continued with steroids and cyclophosphamide. Anti-GBM antibodies became negative and consolidations on the chest x-ray recovered (Fig. 3). Hemoptysis was not evident in the follow-up. He is being followed closely in terms of renal involvement. Although he had pulmonary involvement, he did not have any findings related to renal involvement at the one-year follow-up. Written consent was obtained from the parents.

\section{Discussion}

Goodpasture syndrome is a life threatening, severe autoimmune disease that is dominant in males at early ages. ${ }^{5}$ Although it peaks in third and seventh decades and is uncommon before puberty, our patient was diagnosed when he was 14 years of age. ${ }^{1}$ Isolated pulmonary involvement is very rare in GS and its incidence is not known in childhood. Although the underlying mechanism is the same both in lung and kidney, the disease severity and temporal aspects may change in patients. ${ }^{1}$ The correlation between serum antiGBM antibodies and severity of renal disease is known, but a relationship has not been found in pulmonary diseases. ${ }^{6}$

Several environmental factors were found to be associated with GS, such as viral infections, lithotripsy for renal stones, and exposure to organic solvents; however, none of them were confirmed as the sole cause of the disease. ${ }^{1,7}$ Despite his young age, our patient had a twoyear smoking history and smoking was also reported as one of the possible cause of the disease and lung manifestations. ${ }^{8}$ Smoking may be the cause of early manifestation of pulmonary involvement in this patient.

Minor hemoptysis has several causes and diagnosis is very difficult in such patients without any renal findings. These patients require more invasive procedures such as bronchoscopy for diagnosis. Although acute hemorrhage was not observed during the bronchoscopy and the patient was stable for 18 hours, massive hemoptysis occurred suddenly. There is no data in the literature concerning bronchoscopy and massive hemoptysis in GS. We did not perform any interventional procedure such as a biopsy. However, bronchoalveolar lavage was taken with isotonic saline and was clear, not bloody. It is known that bladder irrigation with normal saline may cause uroepithelial injury which may, in turn, cause inflammation. ${ }^{9}$ We thought that massive hemoptysis may be caused by the same mechanism: Due to lavage with saline and suctioning, inflammation may have been triggered with the occurrence of massive hemoptysis. Renal biopsy was not performed due to rapidly progressive life threatening course, also there were no hematuria, proteinuria or deteriorated renal function tests. Furthermore, the patient did not have any renal finding during the one-year followup.

There are some papers about coexistence systemic lupus erythematosus (SLE) and GS in patients, who had pulmonary haemorrhage. ${ }^{10,11}$ However our case did not exhibit immunological or clinical signs of SLE.

Successful treatment was observed with pulse steroids, cyclophosphamide and plasmapheresis in our patient resulting in antiGBM antibodies becoming negative. During the one-year follow-up, the antibodies did not become positive again. There are no clinical trials for the treatment in GS, but rituximab and mycophenolate mofetil are also reported as treatments. ${ }^{3,12}$

In conclusion, GS should be considered in young men with hemoptysis, particularly those who smoke. Invasive procedures such as bronchoscopy should be avoided as much as possible in making a diagnosis. Furthermore, patients with isolated pulmonary involvement should be followed for renal involvement. 


\section{REFERENCES}

1. Hellmark T, Segelmark M. Diagnosis and classification of Goodpasture's disease (anti- GBM). J Autoimmun 2014; 48-49: 108-112.

2. Huart A, Josse AG, Chauveau D, et al; French Society of Hemapheresis. Outcomes of patients wiyh Goodpasture syndrome: A nationwide cohort-based study from the French Society of Hemapheresis. J Autoimmun 2016; 73: 24-29.

3. Greco A, Rizzo MI, De Virgilio A, et al. Goodpasture's syndrome: A clinical update. Autoimmun Rev 2015; 14: 246-253.

4. Peto P, Salama AD. Update on antiglomerular basement membrane disease. Curr Opin Rheumatol 2011; 23: 32-37.

5. Dammacco F, Battaglia S, Gesualdo L, Racanelli V. Goodpasture's disease: A report of ten cases and a review of the literature. Autoimmun Rev 2013; 12: 1101-1108.

6. Segelmark M, Hellmark T, Wieslander J. The prognostic significance in Goodpasture's disease of specificity, titre and affinity of anti-glomerular basement- membrane antibodies. Nephron Clin Pract 2003; 94: c59-c68.

7. Westman KW, Ericsson UB, Hoier-Madsen M, et al. Prevalence of autoantibodies associated with glomerulonephritis, unaffected after extracorporeal shock wave lithotripsy for renal calculi, in a threeyear follow-up. Scand J Urol Nephrol 1997; 31: 463467.
8. García-Rostan y Pérez GM, García Bragado F, Puras Gil AM. Pulmonary hemorrhage and antiglomerular basement membrane antibody-mediated glomerulonephritis after exposure to smoked cocaine (crack): A case report and review of the literature. Pathol Int 1997; 47: 692-697.

9. Di Paolo M, Bugelli V, Di Luca A, Turillazzi E. Bladder irrigation and urothelium disruption: A reminder apropos of a case of fatal fluid absorption. BMC Urol 2014; 14: 91.

10. Yamazaki K, Fujita J, Doi I, Abe S, Kawakami Y, Fukazawa Y. A case of acute pulmonary hemorrhage and positive anti-glomerular basement membrane antibody in systemic lupus erythematosus. Nihon Kyobu Shikkan Gakkai Zasshi 1993; 31: 251-256.

11. Yamada T, Mugishima K, Higo S, et al. A case of antiglomerular basement membrane antibody-positive systemic lupus erythematosus with pulmonary hemorrhage successfully treated at an early stage of the disease. J Nippon Med Sch 2018; 85: 138-144.

12. García-Cantón C, Toledo A, Palomar R, et al Goodpasture's syndrome treated with mycophenolate mofetil. Nephrol Dial Transplant 2000; 15: 920-922. 